\title{
CNS Actinomycosis presenting with bilateral cavernous sinus syndrome
}
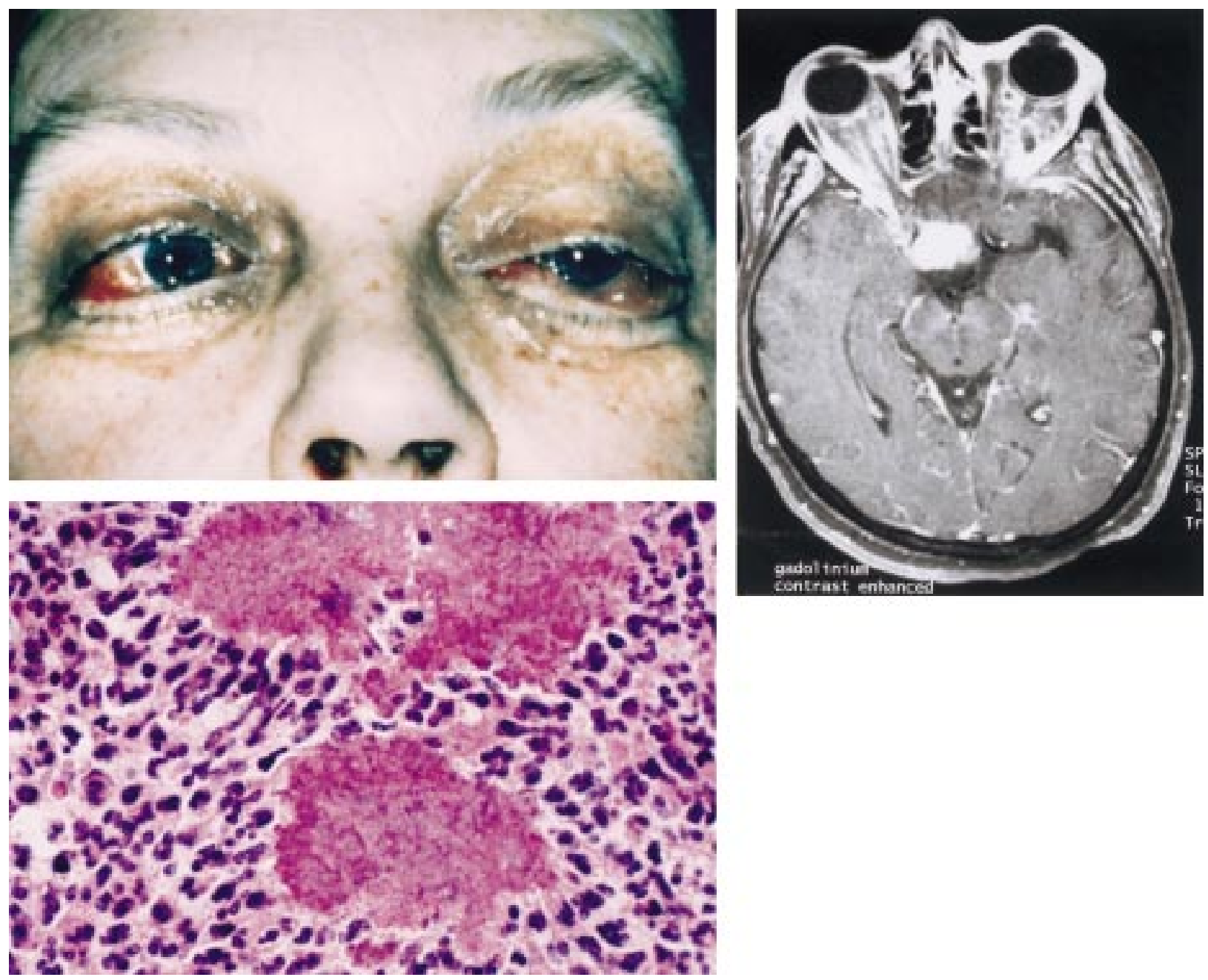

This 47 year old woman presented with subacute bilateral eye redness and pain. She was febrile with signs of meningismus. She had bilateral exophthalmos and chemosis, left ptosis (figure top left), and complete bilateral ophthalmoplegia. Her CSF contained 73 white blood cells $/ \mathrm{mm}^{3}$ (63\% neutrophils) and 7 red blood cells $/ \mathrm{mm}^{3}$, with a protein concentration of $1.3 \mathrm{~g} / 1$ and a glucose concentrationof $0.55 \mathrm{~g} / 1 \quad<10 \%$ of a simultaneous blood glucose concentration). Microbiological studies on the CSF were negative. Cranial MR images showed an enhancing parasellar mass extending into both cavernous sinuses (figure top right). A trans-sphenoidal biopsy of this mass demonstrated acute inflammation with sulfur granules typical of actinomycocis (figure bottom left). Her primary focus was thought to be a dental infection, related to dental caries. She was treated with a prolonged course of high dose intravenous penicillin, and made a complete recovery.

NEIL R HOLLAND ELLEN DEIBERT 